Clinical Genitourinary Cancer, Vol. 16, No. 5, e993-96 a2018 Elsevier Inc. All rights reserved. DOI: https://doi.org/10.1016/i.clgc.2018.05.005

\title{
Case report: Chemotherapy in conjunction with blood-brain barrier disruption for a patient with germ cell tumor with multiple brain
} metastases

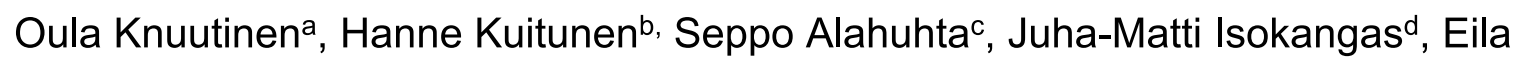
Sonkajärvic, Taina Turpeenniemi-Hujanen ${ }^{b}$, Outi Kuittinen ${ }^{b}$

aMedical Research Center (MRC), University of Oulu, Oulu University Hospital, 90220, Oulu, Finland,

bDepartment of Oncology, MRC, University of Oulu, Oulu University Hospital, 90220, Oulu, Finland,

'Department of Anaesthesiology, MRC, University of Oulu, Oulu University Hospital, 90220, Oulu, Finland,

dDepartment of Diagnostic Radiology, MRC, University of Oulu, Oulu University Hospital, 90220, Oulu, Finland.

The work was carried out at University of Oulu, Finland, and Oulu University Hospital, Finland.

Corresponding author: Oula Knuutinen, Biocenter Oulu, P.O.Box 5000, FI-90014 University of Oulu, Finland. E-mail: oula.knuutinen@student.oulu.fi

Keywords: BBBD therapy, intra-arterial chemotherapy, neuro-oncology, testicular neoplasms

Declarations of interests: none

Funding: This research did not receive any specific grant from funding agencies in the public, commercial, or not-for-profit sectors.

Word count: 1470 (excluding references and figure captions) 


\section{Introduction}

Testicular cancer is a relatively rare malignancy affecting mostly young males of working age. Still, it is the most common solid tumor in men aged $20-34$ years. ${ }^{1,2}$ The incidence of testicular cancer in Finland is 5.8 per 100.000 men which is comparable to that of the United States. ${ }^{3,4}$ The incidence is on the rise since 40 years and is estimated to further increase in the next decade. ${ }^{1,2,5}$

The age-adjusted 5-year relative survival rate of patients with testicular cancer in Finland is $95 \%$ owing to the high sensitivity to chemotherapy. ${ }^{4}$ Although testicular cancer, in general, has an excellent prognosis, the expected 5-year survival of patients with brain metastases (BM) at initial diagnosis is less than $50 \% .^{6,7}$ As surgical treatment and stereotactic radiotherapy are rarely applicable, the most frequently used therapy for BM remains multimodality treatment with chemotherapy and whole brain radiation therapy (WBRT). WBRT is possibly related to survival benefit, but also leads to cognitive decline and long-term neurotoxicity. ${ }^{7,8}$ The most common deleterious cognitive effects are impaired memory and executive function. ${ }^{9} 50-80 \%$ of patients treated with WBRT demonstrate a decline in neurocognitive tests and $12-38 \%$ have impaired Mini-Mental State Examination scores. ${ }^{10}$ Progressive delayed brain toxicity has also been described. ${ }^{11}$

Here we report a case of a patient with BM of testicular cancer treated with chemotherapy in conjunction with blood-brain barrier disruption (BBBD). This promising treatment combines transient disruption of the blood-brain barrier with hyperosmolar intra-arterial 
mannitol infusion and simultaneous intra-arterial administration of chemotherapy. This is known to drastically increase the intracranial and intratumoral concentrations of chemotherapy agents. ${ }^{12,13}$ Treating BM with BBBD therapy, we were able to avoid the deleterious cognitive effects of WBRT with a complete treatment response.

\section{Case report}

A 25-year-old man was admitted to the emergency department of Oulu University Hospital for a headache, visual field loss and fever spanning three days. A week before the admission the patient was assessed for a supraclavicular mass with a diameter of $4 \mathrm{~cm}$ and a fine-needle aspiration was performed. A head MRI revealed 10-15 contrastenhancing tumors in cerebrum and cerebellum in both hemispheres (Fig. 1: 1A-2C). A CT scan of chest, abdomen, and pelvis demonstrated several contrast-enhancing nodules in the lungs and lymphadenopathy in the upper abdomen and mediastinum. Ultrasonography located a primary tumor with a diameter of $13 \mathrm{~mm}$ and irregular borders in the left testicle. Serum and plasma tumor markers $\beta$-HCG $(20.000 \mathrm{U} / \mathrm{l})$ and LDH (805 U/I) were elevated indicating an advanced disease. AFP was within reference values. A radical orchiectomy from inguinal incision was performed within 3 days. The surgical sample demonstrated scar tissue with no viable tumor cells suggesting a regressed tumor. The fine-needle aspiration sample of the supraclavicular mass revealed the final diagnosis of advanced choriocarcinoma and metastases in the brain, lungs, abdomen, and mediastinum.

The patient was treated with four cycles of BEP (bleomycin, etoposide, cisplatin) as recommended by European Consensus Conference on Diagnosis and Treatment of Germ 
Cell Cancer (EGCCCG) report for patients with poor prognosis according to International Germ Cell Consensus Classification Group (IGCCCG). ${ }^{8,14}$ Trends in the tumor marker levels are shown in Fig. 2. After four cycles of PEB, FDG-PET (fluorodeoxyglucose positron emission tomography) scan demonstrated accumulation of FDG in lymph nodes on the neck and para-aortic region and in the pulmonary nodules. The nuchal lymph node was surgically resected for histologic study which revealed a necrotic tumor with no viable tumor cells. However, elevated tumor markers $\beta$-HCG and LDH (128 U/l and $244 \mathrm{U} / \mathrm{l}$, respectively) suggested residual disease. For this reason, a salvage therapy with VIP (etoposide, ifosfamide, cisplatin) was started. After the first VIP, stem cells were collected for potential following high-dose therapy and stem cell transplantation. After the stem cell collection, head MRI revealed four new contrast-enhancing BM in both cerebral hemispheres.

The treatment of choice for multiple BM unresponsive to chemotherapy is WBRT. Considering the patient's young age and long working life expectations the harmful effects of WBRT on cognitive abilities would have been particularly disabling. As our clinic is one of the few centers in the world administering BBBD chemotherapy, the possibility of this experimental treatment was discussed with the patient. He agreed to four cycles of BBBD with a combination of carboplatin, cyclophosphamide, and etoposide - drugs also used in the standard treatment of choriocarcinoma. We have previously described the treatment protocol in detail. ${ }^{15,16}$ Three treatments were administered to each internal carotid artery and one treatment to vertebral arteries. Complete treatment response was observed in MRI after the second cycle. MRI before and after BBBD therapy is shown in Fig. 1. A mild hearing loss caused by platinum-based chemotherapy was noticed in audiogram after the second BBBD therapy. The subsequent doses of carboplatin were reduced, and the 
hearing loss did not progress in 9 months of follow-up. As previously planned, following the BBBD therapy, the patient started a high-dose intravenous chemotherapy with carboplatin and etoposide and underwent autologous stem cell transplantation.

Thirty-two months from the last BBBD therapy, the patient remained in complete remission: the tumor markers were low and imaging studies of brain, chest, abdomen, and pelvis showed no detectable active tumor.

\section{Discussion}

Despite the excellent prognosis of testicular cancer and high sensitivity to chemotherapy, the 5-year survival in patients who develop BM during first-line treatment is less than 5 $\% .{ }^{17}$ In 32 months of follow up, our patient remained in remission. In comparison, the progression-free survival rate of patients with germ cell tumor with brain metastases at initial diagnosis is $30 \%$ at 3 years. ${ }^{7}$ In the case of BM, the blood-brain barrier prevents traditional intravenous drug delivery to tumor tissue. Although the development of surgical treatment and stereotactic radiation therapy has given hope for some patients, these modalities cannot be applied for multiple metastases, and WBRT remains the cornerstone of the treatment. The neurotoxicity of WBRT is especially detrimental for developing children and young adults with long working life expectancy. BBBD therapy is a promising treatment for BM with the potential to avoid the adverse cognitive effects of WBRT.

In addition to BBBD therapy, the patient received high-dose chemotherapy, which might be related to higher long-term survival rates in testicular cancer with BM. ${ }^{1,18}$ However, we find this option doubtful because the radiological response to BBBD was seen before initiating 
high-dose chemotherapy. Moreover, the chemotherapeutic agents used during the highdose therapy (carboplatin and etoposide), are known to have a low penetrance in the CNS. ${ }^{20,21}$ Thus, we believe the sustaining response in the brain and the favorable prognosis seen in our patient is attributable to BBBD therapy. The assessment of the absent cognitive impairment was limited to patient's subjective evaluation of symptoms and no neuropsychological testing was performed. However, McAllister et al. have previously shown in a cohort of patients with primary central nervous system lymphoma that the therapy does not significantly impair cognitive capabilities. ${ }^{22}$

Our article describes a successful treatment of chorionic carcinoma and BM with BBBD therapy. To our knowledge, five cases of patients with germ cell tumor BM treated with BBBD have been reported previously. Two achieved complete response, one partial response and one stable disease evaluated using the MacDonald criteria. ${ }^{21,22}$ Promising results with BBBD have been reported with BM of ovarian carcinoma and adenocarcinomas ${ }^{23}$ and several primary central nervous system tumors including germ cell tumors, ${ }^{24,25}$ primary central nervous system lymphoma, ${ }^{16,24}$ and astrocytomas. ${ }^{26}$ The safety issues with BBBD therapy are similar to any chemotherapy. Most common clinically significant adverse effects are cytopenias and thromboembolic events caused by arterial access. Mortality has been described with only aggressively growing tumors, such as glioblastoma. ${ }^{24}$ Further research is needed to assess the efficacy of BBBD therapy in testicular cancer BM. Still, our study illustrates the opportunity to protect cognitive abilities with good tumor response using BBBD in patients with poor prognosis for whom the treatment options are limited.

\section{Conclusions}


Treatment of testicular cancer with brain metastases is problematic because the conventional intravenous chemotherapy is rendered ineffective due to the blood-brain barrier. This case report demonstrates the use of blood-brain barrier disruption therapy as an alternative to whole brain radiation therapy. With blood-brain barrier disruption therapy, chemotherapy can be effectively delivered to target tumor across the blood-brain barrier.

\section{Clinical Practice Points}

- Testicular cancer with brain metastases is related to poor prognosis because the penetration of chemotherapeutic agents is decreased by the blood-brain barrier.

- The standard treatment of brain metastases - whole brain radiation therapy combined with chemotherapy - is related to a limited increase in survival and considerable deleterious cognitive effects.

- The blood-brain barrier can be transiently disrupted using hyperosmolar intraarterial mannitol injection. When combined with intra-arterial chemotherapy, therapeutic intratumoral concentrations can be attained.

- In experienced centers, blood-brain barrier disruption (BBBD) therapy is relatively safe with a low incidence of catheter-related complications.

- BBBD therapy is a promising treatment modality for brain metastases as an alternative to whole brain radiation therapy. 


\section{References}

1. Ghazarian AA, Kelly SP, Altekruse SF, Rosenberg PS, McGlynn KA. Future of testicular germ cell tumor incidence in the United States: Forecast through 2026. Cancer. 2017;123(12):2320-2328. doi:10.1002/cncr.30597.

2. Richiardi L, Bellocco R, Adami H-O, et al. Testicular cancer incidence in eight northern European countries: secular and recent trends. Cancer Epidemiol Biomarkers Prev. 2004;13(12):2157-2166.

http://www.ncbi.nlm.nih.gov/pubmed/15598775.

3. Siegel RL, Miller KD, Jemal A. Cancer statistics, 2018. CA Cancer J Clin. 2018;68(1):7-30. doi: 10.3322/caac.21442

4. Finnish Cancer Registry. Finnish Cancer Statistics. https://syoparekisteri.fi/tilastot/tautitilastot/. Accessed October 2, 2017.

5. McGlynn KA, Devesa SS, Sigurdson AJ, Brown LM, Tsao L, Tarone RE. Trends in the incidence of testicular germ cell tumors in the United States. Cancer. 2003;97(1):63-70. doi:10.1002/cncr.11054.

6. Krege S, Beyer J, Souchon R, et al. European Consensus Conference on Diagnosis and Treatment of Germ Cell Cancer: A Report of the Second Meeting of the European Germ Cell Cancer Consensus Group (EGCCCG): Part I. Eur Urol. 2008;53(3):478-496. doi:10.1016/j.eururo.2007.12.024

7. Feldman DR, Lorch A, Kramar A, et al. Brain Metastases in Patients With Germ Cell Tumors: Prognostic Factors and Treatment Options-An Analysis From the Global Germ Cell Cancer Group. J Clin Oncol. 2016. doi:10.1200/JCO.2015.62.7000.

8. Krege S, Beyer J, Souchon R, et al. European Consensus Conference on Diagnosis and Treatment of Germ Cell Cancer: A Report of the Second Meeting of the 
European Germ Cell Cancer Consensus Group (EGCCCG): Part II. Eur Urol. 2008;53(3):497-513. doi:10.1016/j.eururo.2007.12.025.

9. McDuff SGR, Taich ZJ, Lawson JD, et al. Neurocognitive assessment following whole brain radiation therapy and radiosurgery for patients with cerebral metastases: Table 1. J Neurol Neurosurg Psychiatry. 2013;84(12):1384-1391. doi:10.1136/jnnp2013-305166.

10. Tallet A V, Azria D, Barlesi F, et al. Neurocognitive function impairment after whole brain radiotherapy for brain metastases: actual assessment. Radiat Oncol. 2012;7(1):77. doi:10.1186/1748-717X-7-77.

11. Doyle DM, Einhorn LH. Delayed Effects of Whole Brain Radiotherapy in Germ Cell Tumor Patients with Central Nervous System Metastases. Int J Radiat Oncol. 2008;70(5):1361-1364. doi:10.1016/J.IJROBP.2007.11.005.

12. Morikawa N, Mori T, Abe T, Kawashima H, Takeyama M, Hori S. Pharmacokinetics of Etoposide and Carboplatin in Cerebrospinal Fluid and Plasma during Hyperosmotic Disruption of the Blood Brain Barrier and Intraarterial Combination Chemotherapy. Biol Pharm Bull. 1999;22(4):428-431. doi:10.1248/bpb.22.428.

13. Zylber-Katz E, Gomori JM, Schwartz A, Lossos A, Bokstein F, Siegal T. Pharmacokinetics of methotrexate in cerebrospinal fluid and serum after osmotic blood-brain barrier disruption in patients with brain lymphoma. Clin Pharmacol Ther. 2000;67(6):631-641. doi:10.1067/mcp.2000.106932.

14. IGCCCG. International Germ Cell Consensus Classification: a prognostic factorbased staging system for metastatic germ cell cancers. International Germ Cell Cancer Collaborative Group. J Clin Oncol. 1997;15(2):594-603. doi:10.1200/JCO.1997.15.2.594. 
15. Kiviniemi V, Korhonen V, Kortelainen J, et al. Real-time monitoring of human bloodbrain barrier disruption. Janigro D, ed. PLoS One. 2017;12(3):e0174072. doi:10.1371/journal.pone.0174072.

16. Kuitunen $\mathrm{H}$, Tokola S, Siniluoto $\mathrm{T}$, et al. Promising treatment results with blood brain barrier disruption (BBBD) based immunochemotherapy combined with autologous stem cell transplantation (ASCT) in patients with primary central nervous system lymphoma (PCNSL). J Neurooncol. 2017;131(2):293-300. doi:10.1007/s11060-0162293-8.

17. Spears WT, Morphis JG, Lester SG, Williams SD, Einhorn LH. Brain metastases and testicular tumors: Iong-term survival. Int J Radiat Oncol Biol Phys. 1992;22(1):17-22. doi: 10.1016/0360-3016(92)90977-P.

18. Oechsle K, Kollmannsberger C, Honecker F, Boehlke I, Bokemeyer C. Cerebral metastases in non-seminomatous germ cell tumour patients undergoing primary high-dose chemotherapy. Eur J Cancer. 2008;44(12):1663-1669. doi:10.1016/J.EJCA.2008.05.012.

19. Kollmannsberger C, Nichols C, Bamberg M, et al. First-line high-dose chemotherapy \pm radiation therapy in patients with metastatic germ-cell cancer and brain metastases. Ann Oncol. 2000;11:553-559. doi:10.1023/A:1008388328809

20. Kiya K, Uozumi T, Ogasawara H, et al. Penetration of etoposide into human malignant brain tumors after intravenous and oral administration. Cancer Chemother Pharmacol. 1992;29(5):339-342. doi:10.1007/BF00686001.

21. Jacobs S, McCully CL, Murphy RF, Bacher J, Balis FM, Fox E. Extracellular fluid concentrations of cisplatin, carboplatin, and oxaliplatin in brain, muscle, and blood measured using microdialysis in nonhuman primates. Cancer Chemother Pharmacol. 2010;65(5):817-824. doi:10.1007/s00280-009-1085-7. 
22. McAllister LD, Doolittle ND, Guastadisegni PE, et al. Cognitive Outcomes and Longterm Follow-up Results after Enhanced Chemotherapy Delivery for Primary Central Nervous System Lymphoma. Neurosurgery. 2000;46(1):51-61. doi:10.1093/neurosurgery/46.1.51

23. Fortin D, Gendron C, Boudrias M, Garant MP. Enhanced chemotherapy delivery by intraarterial infusion and blood-brain barrier disruption in the treatment of cerebral metastasis. Cancer. 2007;109(4):751-760. doi:10.1002/cncr.22450.

24. Doolittle ND, Miner ME, Hall W a, et al. Safety and efficacy of a multicenter study using intraarterial chemotherapy in conjunction with osmotic opening of the bloodbrain barrier for the treatment of patients with malignant brain tumors. Cancer. 2000;88(3):637-647. doi: 10.1002/(SICI)1097-0142(20000201)88:3<637::AIDCNCR22>3.0.CO;2-Y.

25. Jahnke K, Kraemer DF, Knight KR, et al. Intraarterial chemotherapy and osmotic blood-brain barrier disruption for patients with embryonal and germ cell tumors of the central nervous system. Cancer. 2008;112(3):581-588. doi:10.1002/cncr.23221.

26. Guillaume DJ, Doolittle ND, Gahramanov S, Hedrick NA, Delashaw JB, Neuwelt EA. Intra-arterial chemotherapy with osmotic blood-brain barrier disruption for aggressive oligodendroglial tumors: Results of a phase i study. Neurosurgery. 2010;66(1):48-58. doi:10.1227/01.NEU.0000363152.37594.F7. 
Figure Captions (figures in black and white, not in color)

Fig. 1. MR imaging of the brain before and after BBBD therapy. Above are T1-weighted (1A-C) and susceptibility-weighted angiography (SWAN) (2A-C) sequence images after PEB therapy. Several lesions with a low T1 signal are seen in both hemispheres of the cerebrum and cerebellum $(1 \mathrm{~A}-\mathrm{C})$. Some show contrast enhancement (1B, arrows). Abnormalities in SWAN (2A-C) correlate with hemosiderin accumulation and bleeding. There is a larger lesion with a hematoma in the left occipital lobe (1B and 2B, arrowhead). After BBBD therapy, the metastatic lesions no longer display contrast enhancement. The hematoma has reduced in size (2B, arrowhead). The signal abnormalities in SWAN sequence remained unchanged (not shown).

Fig. 2. Trends of tumor markers.

After orchiectomy and four cycles of PEB chemotherapy, tumor markers remained elevated and new brain lesions were noticed. To achieve systemic tumor control, highdose chemotherapy was planned with stem cell mobilization with VIP. Four treatments with BBBD therapy were administered achieving a complete response. $\beta-H C G$ and LDH have remained stable with no clinical signs of relapse. 


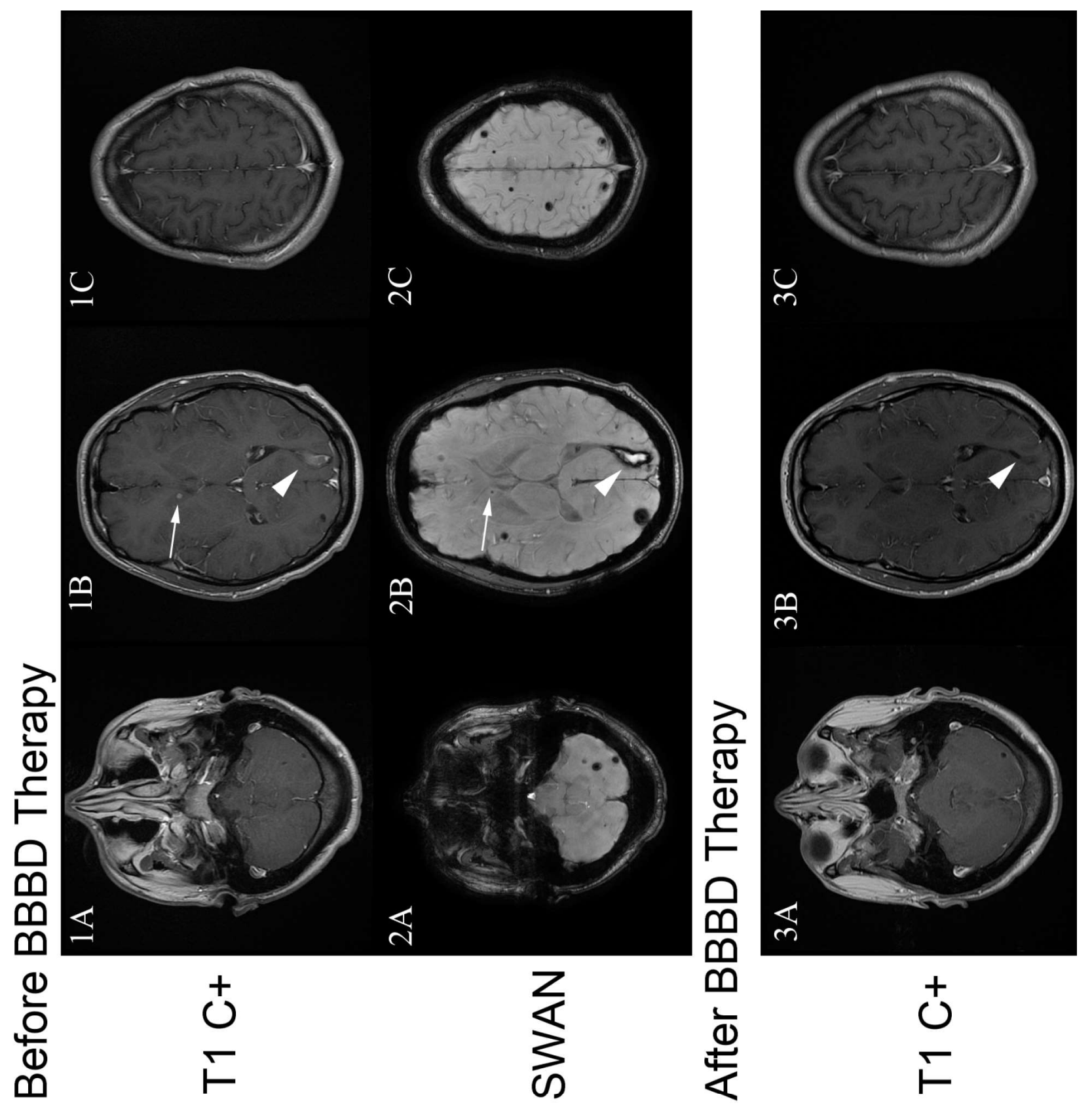


$[/ / \mathrm{N}] \mathrm{HO}$

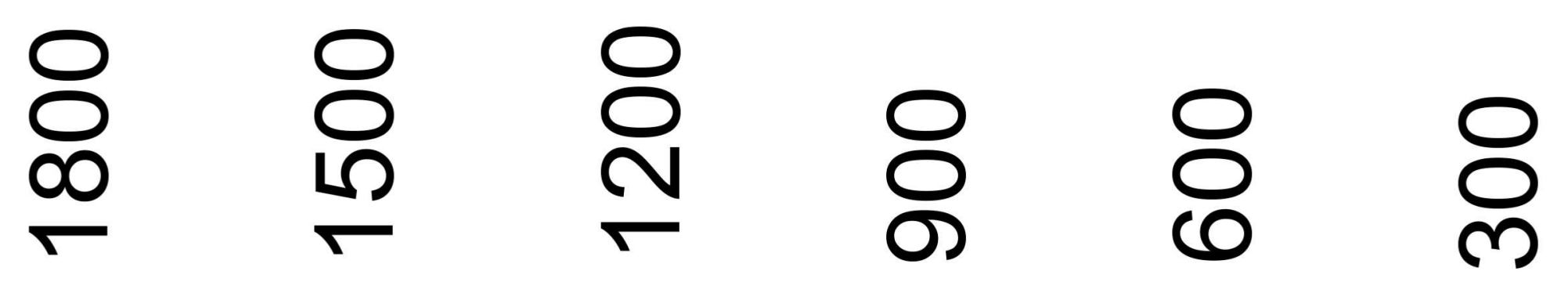

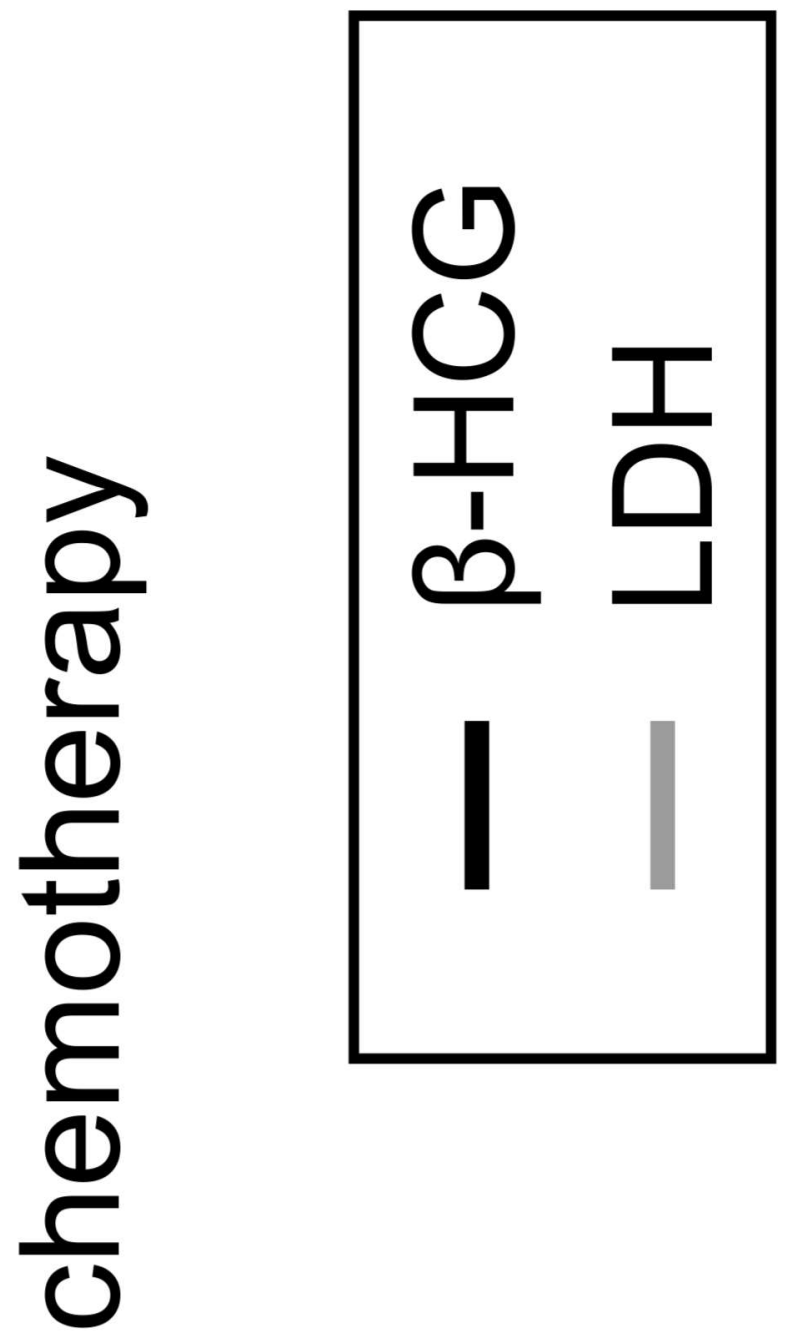

(1)

क

응

$\frac{1}{\frac{1}{c}}$ 